\title{
Presentación del Dossier. Pierre Bourdieu: trabajo sociológico e investigación en las ciencias sociales y las humanidades
}

\author{
Dossier Presentation. Pierre Bourdieu: Sociological work and research in the social sciences and humanities
}

\section{Armando Ulises Cerón Martínez}

Universidad Autónoma del Estado de Hidalgo - México

Pachuca, México

aceron@uaeh.edu.mx

\section{Eva María Galán Mireles}

Universidad Autónoma del Estado de Hidalgo - México

Pachuca, México

eva.galan.mireles@gmail.com

\section{Presentación}

¿Cuáles son los retos que implica adherirse a una cultura teórica en una investigación científica? Sea consciente o no, el científico social opera bajo un paradigma científico y, por lo tanto, bajo un esquema teórico y metodológico particular, pero se es más eficiente cuando se cobra conciencia de ello. Esta práctica consciente es una actividad reflexiva, muy propia de esa misión que Pierre Bourdieu asignó a la práctica sociológica.

En este sentido, conviene clarificar que la práctica sociológica no es privativa para el sociólogo formal, el que adquirió su título profesional por su carrera, sino que sociólogo es todo aquel interesado en investigar problemas o fenómenos sociales con herramientas científicas. Esta es una de las razones por las que cualquiera que elige la propuesta teórica de Pierre Bourdieu para su investigación puede aplicarla a diversos terrenos empíricos: la fotografía, los museos, los cómics, la medicina, el deporte, la educación, la religión, la moda, la cultura, la vivienda, etc.

Esto debía alertarnos sobre los múltiples objetos de la ciencia que es posible abordar desde las propuestas de Pierre Bourdieu para la investigación científica. Al respecto, el autor consideró que sus tres conceptos nodales (Campo, Capital y Habitus) debían ser puestos en operación de forma conjunta porque en ellos hay una complementación de dos tradiciones opuestas: objetivismo y subjetivismo. Las condiciones sociales objetivas pueden ser aprehendidas por medio del análisis sistemático de las nociones de campo y de capital, pues son entidades concretas, asibles por su distribución estadística en el espacio social, en tanto que ajenas a los voluntarismos ingenuos. Las condiciones sociales subjetivas (formas de pensar, de sentir, de opinar, etc.), son captadas bajo la noción de habitus en tanto que “... sistema de disposiciones duraderas y transferibles, estructuras estructuradas predispuestas a funcionar como estructuras estructurantes..." (Bourdieu 2007, p. 86).

El habitus científico de Bourdieu se movió en los tres niveles de la lógica de la ciencia que Michael Polanyi propuso (en Bourdieu, Chamboderon \& Passeron, 2002, p.50): los objetos de la ciencia, la ciencia misma y la metaciencia. De esta manera, los trabajos que aquí se presentan abordan estos tres niveles y, por lo tanto, son igualmente válidos para ser pensados como trabajos propiamente bourdianos, además de ser presentados en el orden de prioridad de las nociones de campo, capital y habitus, según lo abordan los trabajos.

Bajo este entendido, la presentación de los artículos que integran el presente dossier comienza con el trabajo denominado El campo del pensamiento decolonial latinoamericano, de Adrián Galindo, el cual 
tiene como objetivo, a partir de la teoría de los campos de Bourdieu, desarrollar qué es un análisis con características empacipadoras desde los autores que comenzaron a desarrollar el término decolonial, así como las etapas que por las que ha transitado esta perspectiva. Este trabajo establece tres aspectos de análisis: la relación que guarda el pensamiento decolonial con el legado sociológico occidental, el uso particular el término epistemología, y el papel de las instituciones universitarias en la producción de conocimiento.

En La construcción del campo científico universitario y sus reflejos en la producción del Homo Academicus de Pierre Bourdieu, de Catarina Gomes, se confirma que las relaciones existentes entre un campo y el campo del poder establecen las reglas del juego para los agentes involucrados e interesados en ser parte de él, al fomentar la producción y la reproducción de prácticas científicas en forma de habitus, sin establecer por ello una estructura finita por las mismas dinámicas que se ajustan a las posiciones ocupadas dentro del campo, ya como dominados o dominantes. Finalmente, se puede detectar de manera dialógica la tensión entre la ortodoxia y la heterodoxia que puede se explícita o implícita, y tiende a ejercerse de manera pasiva o a través de la violencia simbólica.

El trabajo de Sarahi Isuki Castelli Olvera, El campo y la historieta mexicana de fin de siglo, se enfoca en la interacción de consumo en el campo de las historietas, en específico, de las décadas de los ochenta y noventa, para después identificar los diversos agentes y sus posturas que finalmente permitieran examinar los ejercicios de poder y de resistencia que incluyen la historieta industrial y la historieta contracultural. Este trabajo utilizó una metodología documental a través de un análisis de las colecciones particulares de las décadas antes mencionadas para desarrollar una construcción del campo, los jugadores, las posiciones, las reglas del juego, etc., entre otros conceptos de Pierre Bourdieu que pueden ser utilizados como herramientas y objetos de estudio.

El caso Los Novísimos: una revolución simbólica específica en la génesis del campo artístico paraguayo, de Sergio Rojas, hace una propuesta de imaginación sociológica que incluye orden epistemológico y metodológico, al realizar un cruce entre lo teórico y lo empírico entre casos históricos a partir de la pregunta ¿qué se tiene entre manos cuando un objeto presenta propiedades que parecen fundamentar, en unas condiciones espaciotemporales distintas a las que correspondían al caso francés, la transposición de un modelo teórico como el de la "génesis del campo artístico"?, con el propósito de comprender relacionalmente las propiedades distintivas y específicas de las acciones de los agentes, por lo que fue necesario hacerlo a partir de la comprensión y el surgimiento del campo, construyendo su estructura, e identificando las posiciones de los agentes y las instituciones para calcular su relativa importancia, especialmente en términos de capital simbólico.

Producción de Bienes Simbólicos en el Campo Museal en Colombia: Reconfiguración de la narrativa de estado con Acuerdo de Paz, de Clavijo, Ospina y Sánchez, tiene como propósito analizar estos espacios culturales, entendidos como sitios en los cuales se produce y reproduce el discurso oficial del estado. Tiene como base las nociones de campo, capital y bienes simbólicos de Pierre Bourdieu. Este trabajo parte de la revisión documental de orden analítico que permitió confeccionar la caracterización del Campo Museal en Colombia. Además, conformó la percepción y los ajustes sobre los actores construidos desde y para el Estado que en palabras de Bourdieu (2014), el Estado ha de ser considerado como "un punto de vista con vista a todos los puntos de vista, el cual no es más un punto de vista ya que, es con relación a él que todos los puntos de vista están organizados" (p.5). Todo lo anterior es posible a partir de la producción de bienes simbólicos en los museos y de la firma del Acuerdo Final para comprender la posición e influencia que tienen los museos en el campo artístico y el campo de poder.

En Efecto de lugar: aportes para comprender la segmentación socioeducativa en dos espacios locales de Argentina, la Dra. Sassera dice que "La obra de Pierre Bourdieu es extensa y contribuye a la comprensión de múltiples fenómenos sociales". En este caso particular, la noción se utiliza como una herramienta analítica para explorar el vínculo entre desigualdades espaciales y desigualdades educativas con relación al acceso de la población a las instituciones educativas diferenciadas que dan lugar a la segmentación socioeducativa, reconstruida desde la noción de dimensión espacial, entendida ésta como la conformación de grupos o circuitos escolares, en específico, de las ciudades de Campana y Zárate (provincias de Buenos Aires, Argentina) de escuelas secundarias, secundarias técnicas e instituciones de educación y formación para el trabajo que presentan características similares a los que acceden los diferentes sectores sociales. Este proyecto se realizó a partir de la aplicación de entrevistas semi estructuradas a directores, docentes y estudiantes de una muestra de escuelas secundarias y secundarias técnicas de ambas localidades, lo que permitió la obtención de resultados que revelan las diferencias entre las clases o grupo sociales. 
Sara Bravo presenta un trabajo denominado Lucha de poder en la reestructuración del posgrado en pedagogía, Universidad Nacional Autónoma de México 1996-1998, que focaliza el papel de los ajustes del campo académico a partir de las reformas al Reglamento General de Estudios de Posgrado de 1996 en la Universidad Nacional Autónoma de México (UNAM), como producto de las tensiones de fuerza entre los agentes activos. La metodología empleada fue de tipo documental y con entrevistas a informantes clave elegidos en función de los capitales detentados.

Luis Arturo Guerrero en Capitales, habitus y disposiciones de profesores universitarios. Una aproximación a partir de sus trayectorias académicas, aborda los tres momentos propuestos por Pierre Bourdieu para el análisis de los campos: la relación del campo frente al campo de poder, detección de las posiciones en el campo y el análisis de los habitus, articulados por técnicas de carácter relacional como el análisis de correspondencias múltiples (ACM) y el análisis comparado, dotándoles del contenido empírico correspondiente.

Aproximación a la incorporación del capital digital en la escuela de Carlos George y Lilian Salado remite a una discusión particular sobre lo que denominan "capital digital" con relación a las Tecnologías de la Información y la Comunicación (TIC). Con un análisis documental que utiliza la cartografía conceptual, se desprenden ocho categorías analíticas que permitieron el abordaje del problema. Concluyen los autores que "la incorporación de las herramientas tecnológicas en los sistemas escolares no representa alcanzar una mayor igualdad en el campo educativo, sino que está determinado por diversos factores como la posesión de capitales que proporciona una forma diferente de medir la desigualdad en la distribución de los recursos tecnológicos y saberes digitales que están presentes en la institución escolar".

Brenda Rico en El habitus que conformó a Bourdieu: entre capitales y campos de poder, aplica un socioanálisis partiendo de la idea de que "los pensadores sociales deben ser comprendidos como sujetos históricos" (Wood, 2011, p. 16), y Pierre Bourdieu es su objeto de reflexión. En este sentido, Pierre Bourdieu es objeto de estudio y su propio pensamiento es la herramienta para ello. Con detalles de biografía personal y académica, se da cuenta de cómo se fue conformando el filósofo universitario que fue en un etnólogo y de ahí en sociólogo.

Marc Joly y Sergio Sandoval parten de la pregunta hecha artículo ¿Pierre Bourdieu era "bachelardiano"? para desarrollar la propuesta que responder afirmativamente a la pregunta es delimitar los desarrollos ulteriores de Bourdieu. El análisis del trabajo parte de la idea de que si bien siempre hay referencia del sociólogo bearnés a la epistemología de Gaston Bachelard, ello mismo es un límite que no permite comprender la noción de "reflexividad" del primero. Los interlocutores base para tal discusión son Jean Claude Passeron y Denis Baranger con sus propuestas de la adhesión de Bourdieu a la perspectiva bachelardiana.

Ernesto Santillán en Bourdieu: El impacto de una palabra en la investigación de las juventudes, basado en el texto de 1978 La juventud no es más que una palabra, del sociólogo francés, parte de la idea de que a pesar de la distancia en el tiempo del texto, sus postulados siguen aún vigentes en cuanto al estudio de las juventudes, donde la noción de "joven" es resultado de arbitrarias luchas intergeneracionales, pero donde el habitus juvenil garantiza la permanencia de las estructuras sociales al estar incorporadas, y donde el espacio escolar es el sitio donde se naturaliza la condición de ser "joven".

De este modo, este dossier es una invitación a pensar la investigación social desde la perspectiva propiamente bourdiana en tanto que habitus científico siempre inacabado, pero siempre en construcción, siempre orientador y siempre polémico. Las nociones de campo, capital y habitus sólo pueden mostrar su potencial heurísitico cuando son usados en la investigación empírica, razón por la que este número ha privilegiado este tipo de trabajos sin excluir por ello las discusiones metacientíficas.

Editores Invitados

Pachuca, México

25 de septiembre de 2020

\section{REFERENCIAS}

Bourdieu, P. (2007). El sentido práctico. Siglo XXI Editores.

Bourdieu, P. (2014). On the State. Lectures at the College de France 1989-1992. Polity Press.

Bourdieu, P., Chamboderon, J. C. \& Passeron, J. C. (2002). El oficio de sociólogo. Presupuestos epistemológicos. Siglo XXI Editores.

Wood, E. M. (2011). De ciudadanos a señores feudales. Paidós. 